\title{
Altered expression of E-cadherin in breast cancer: patterns, mechanisms and clinical significance
}

\author{
K.S. Ásgeirsson ${ }^{\mathrm{a}}$, J.G. Jónasson ${ }^{\mathrm{b}}$, L. Tryggvadóttir ${ }^{\mathrm{c}}$, K. Ólafsdóttir ${ }^{\mathrm{b}}$, \\ J.R. Sigurgeirsdóttir ${ }^{\mathrm{b}}$, S. Ingvarsson ${ }^{\mathrm{b}}$, H.M. Ögmundsdóttir ${ }^{\mathrm{a}, *}$ \\ ${ }^{a}$ Molecular and Cell Biology Laboratory, Icelandic Cancer Society, PO Box 5420, Reykjavík, Iceland \\ ${ }^{\mathrm{b}}$ Department of Pathology, National Hospital, Reykjavik, Iceland \\ 'Icelandic Cancer Registry, Icelandic Cancer Society, Reykjavik, Iceland
}

Received 19 July 1999; received in revised form 11 January 2000; accepted 2 March 2000

\begin{abstract}
Reduced cell adhesion brought about by altered surface expression of E-cadherin has been implicated in invasive and metastatic malignant growth. We investigated the patterns of immunohistochemical E-cadherin expression in 120 breast carcinomas. Furthermore, we analysed DNA from the same samples for loss of heterozygosity (LOH) using three separate microsatellite markers on chromosome 16q22.1. Finally, the clinical outcome was ascertained for 108 patients. 19\% (18/97) of infiltrating ductal carcinomas showed complete loss of E-cadherin expression compared with 64\% (9/14) of infiltrating lobular carcinomas. LOH was detected in $46 \%(24 / 52)$ of infiltrating ductal carcinomas and $89 \%(8 / 9)$ of infiltrating lobular carcinomas. In the infiltrating lobular carcinomas, LOH was associated with complete loss of cell membrane expression of E-cadherin, although a cytoplasmic expression pattern was evident. In contrast, this association was not seen in the infiltrating ductal carcinomas. In a multivariate analysis, loss of Ecadherin expression was shown to be a significant independent risk factor for a poorer disease-free survival $(P=0.019)$, in particular in the node-negative subset of patients $(P=0.029)$. Significance was also approached for breast cancer corrected survival $(P=0.056)$. We conclude that different mechanisms are involved in the altered E-cadherin expression seen in different subtypes of breast carcinomas. Furthermore, we implicate loss of E-cadherin, regardless of the genetic causes, as an independent prognostic marker for disease recurrence, especially in node-negative breast cancer patients, irrespective of the histological type. (C) 2000 Elsevier Science Ltd. All rights reserved.
\end{abstract}

Keywords: Breast cancer; E-cadherin; Prognosis; Node-negative breast cancer

\section{Introduction}

The development of malignant tumours is in part characterised by alterations in cell-cell adhesion of the tumour cells and their ability to grow invasively into surrounding tissue [1]. In vitro studies have shown that the $\mathrm{Ca}^{2+}$-dependent adhesion molecule, E-cadherin, which is expressed on the surface membranes of epithelial cells, plays a crucial anti-invasive role [2]. When Ecadherin antibodies are added to the medium of MDCK epithelial cells, a decrease in intercellular adhesion and an increase in invasiveness becomes evident [3]. Downregulation of E-cadherin expression of these cells by sarcoma virus transformation results in an invasive

* Corresponding author. Tel.: + 354-540-1909; fax: + 354-5401905.

E-mail address: helgam@krabb.is (H.M. Ögmundsdóttir). phenotype [4]. Furthermore, studies on cell lines derived from various carcinomas have shown that decreased Ecadherin expression is associated with dedifferentiation, invasiveness of epithelial cells and formation of metastases [4-6]. A study in transgenic mice has even suggested that loss of E-cadherin-mediated cell adhesion may play a causal role in the progression from benign adenoma to malignant carcinoma [7]. The implication of E-cadherin in carcinogenesis is supported by the fact that alterations in its expression are frequently seen in various carcinomas including breast cancer [8-12]. The clinical significance of these findings varies between different types of carcinomas, although an association between decreased E-cadherin expression and shortened disease-free survival and poor prognosis has been observed in various malignancies such as renal cell carcinoma, gastric carcinoma and bladder carcinoma [13$16]$. 
For breast cancer, only a few studies have addressed the long-term prognostic effects of altered E-cadherin expression and most of them have indicated an association between altered E-cadherin expression and decreased overall survival $[8,17-19]$. In one of these studies this association was restricted to axillary node-negative breast cancer patients [18].

The mechanisms leading to alterations in E-cadherin expression are poorly understood. These may include mutations in the E-cadherin gene, post-translational modifications of E-cadherin and abnormal function of the normally expressed E-cadherin molecule because of alterations in its associated proteins [20-23]. In vitro studies have shown that when the cytokine IL-6 was added to the medium of three breast cancer cell lines, decreased cell adhesion became evident several days later and this was associated with a decrease in Ecadherin expression of these cells [24]. Furthermore we found that IL-6 serum levels were raised in a significant number of breast cancer patients and that E-cadherin expression of their tumours was often altered, although a direct association could not be established.

In the present study, we examined the pattern of immunohistochemical expression of E-cadherin in paraffin sections from 120 primary breast carcinomas and 37 lymph node metastases. In an attempt to elucidate better the mechanisms by which altered E-cadherin expression occurs we analysed DNA from the same samples for loss of heterozygosity $(\mathrm{LOH})$ of the Ecadherin gene using three microsatellite markers located on chromosome 16q22.1. Furthermore, we did a mutational analysis on four lobular breast carcinomas, two of which showed a cytoplasmic E-cadherin expression pattern on immunohistochemistry and $\mathrm{LOH}$ on polymerase chain reaction (PCR). Finally, we evaluated the clinical significance of our findings.

\section{Patients and methods}

\subsection{Tumour specimens and patients}

Paraffin sections from 120 non-selected primary breast tumours and 37 lymph node metastases were collected from patients undergoing surgery over a 4year period, between January 1990 and September 1994. These tumours were kindly supplied by the Dungal's sample collection, Department of Pathology, University of Iceland.

Information on tumour size, lymph node metastases at diagnosis and oestrogen receptor expression was made available from the Department of Pathology, National University Hospital of Iceland and the Icelandic Cancer Registry. Patient registries from the St Joseph's Hospital in Hafnarfjördur, the City Hospital and the National Hospital in Reykjavík were evaluated for information on TNM staging, disease recurrence, metastases development and survival of patients. Additional information on the present disease status was kindly supplied by the patients' oncologists if information in the hospital records was not sufficient.

\subsection{Immunohistochemical staining}

E-cadherin expression was detected by the $5 \mathrm{H} 9$ monoclonal antibody (kindly donated by Professor Walter Birchmeier and Jürgen Behrens, Max-DelbrückCentrum for Molecular Medicine, Berlin, Germany). Paraffin-embedded $4 \mu \mathrm{m}$ tumour sections were placed on super frost/plus slides from Menzel-Gläser and dried in an oven at $60^{\circ} \mathrm{C}$ for $60 \mathrm{~min}$ and then at $37^{\circ} \mathrm{C}$ for $24 \mathrm{~h}$. Thereafter sections were deparaffinised, rehydrated and rinsed in tap water before antigen retrieval by heating in a $0.01 \mathrm{M}$ citric buffer $(\mathrm{pH} 6.0)$ twice for $5 \mathrm{~min}$ in a Toshiba microwave oven (ER-6E2W) at $850 \mathrm{~W}$. Sections were incubated with $5 \mathrm{H} 9$ monoclonal antibody (1:10) overnight at room temperature. Immunohistochemical staining was visualised, using the StreptABComplex/horse radish peroxidase (HRP) Duet, Mouse/ Rabbit (1:100) (from DAKO, Glostrup, Denmark) according to the manufacturer's instructions.

\subsection{Immunohistochemical analysis}

E-cadherin expression in a fibroadenoma was used as a positive control. The status of E-cadherin expression was evaluated independently by three of the authors. If any discrepancies between classification of samples arose, they were reviewed and the final result was reached by consensus. Tumours were classified by intensity of staining and the percentage of cells showing loss of E-cadherin expression. These two parameters were then combined into a final classification of staining as follows (Table 1): positive, where staining was similar to the positive control sample; heterogeneous, staining not as intense as the positive control and tumours showing a mixed population of E-cadherin-positive and -negative cells; negative, where most or all tumour cells showed no staining or where cytoplasmic as opposed to membrane staining was observed.

\subsection{DNA extraction and analysis}

It was possible to extract DNA from 86 breast carcinomas and peripheral blood using conventional phenol/ chloroform methods. The DNA was subjected to PCR amplification using DynaZyme polymerase (Finnzymes Oy, Espoo, Finland) in the buffer solution provided by the manufacturer.

The PCR amplification was carried out in $25 \mu 1$ reaction volumes in 96-well plates (Techne, Cambridge, $\mathrm{UK}$ ), using $25 \mathrm{ng}$ of genomic DNA, $1.8 \mathrm{pmol}$ of the 
Table 1

Classification of tumours according to E-cadherin expression

$\%$ Cells showing loss of E-cadherin expression

$>50 \quad 5-50 \quad<5$

Intensity of staining

\begin{tabular}{|c|c|c|c|}
\hline- & Negative & & \\
\hline$+/-$ & Heterogeneous & Heterogeneous & Heterogeneous \\
\hline++ & Heterogeneous & Positive & Positive \\
\hline
\end{tabular}

forward and reverse primers, $3.3 \mathrm{nmol}$ of each dNTP and 0.67 units of DynaZyme polymerase. A hot start was used by adding the enzyme during the first cycle at approximately $72^{\circ} \mathrm{C}$, after pre-incubation at $94^{\circ} \mathrm{C}$ for 5 min. The samples were amplified in 35 cycles composed of $30 \mathrm{~s}$ of denaturation at $94^{\circ} \mathrm{C}, 30 \mathrm{~s}$ annealing at $55^{\circ} \mathrm{C}$ and finally $60 \mathrm{~s}$ of extension at $72^{\circ} \mathrm{C}$. Three microsatellite markers were used; D16S421 (forward: 5'ACATGAACCGATTGGACTGA-3', reverse: $5^{\prime}$ CCGTTCCCTATATTTCCTGG-3'), D16S496 (forward: 5'-GAAAGGCTACT-TCATAGATGGCAAT3'), reverse: 5'-ATAAGCCACTGCGCCCAT-3') (both from Research Genetics, Huntsville, AL, USA) and D16S545 (which was kindly donated by Anne-Marie Cleton (Department of Human and Clinical Genetics, Leiden, The Netherlands), forward: 5'-ACTTGAAATGCAGAGTCCAGAA-3', reverse: 5'-TTGCCTGGAGCACATTAGC-3').

The primers for SSCP (single-strand conformation polymorphism) and DNA sequencing of genomic DNA by the solid phase method were according to Berx and colleagues [21]. All reverse primers were biotinylated. The PCR products were immobilised on solid support using streptavidin beads (M-280/Dynabeads, Dynal, Oslo, Norway) and denatured with alkaline. The DNA template was sequenced using sequenase and $\left[\alpha^{33} \mathrm{P}\right]$ dATP (Amersham, Aylesbury, UK). Amplification for the sequencing and SSCP analyses of exon 13 were carried out using the following primers at an annealing temperature of $60^{\circ} \mathrm{C}$ : forward: 5'-TTTCCTCCCCTGGTCTCATC-3'; reverse: 5'-TGAGTCACTTGCCAGCTGGA-3'.

The PCR products were subjected to denaturing gel electrophoresis in $6.5 \%$ polyacrylamide, $8 \mathrm{M}$ urea denaturing gel for $\mathrm{LOH}$ analysis or denatured in a formamide buffer and subjected to a non-denaturing polyacrylamide gel (MDE (mutation detection enhancement, Rockland, Maine, USA) solution/FMC BioProducts) for SSCP analysis. They were then transferred to a positively-charged nylon membrane, Hybond- ${ }^{+}$ (Amersham) and baked for at least $2 \mathrm{~h}$ at $80^{\circ} \mathrm{C}$. Films were inspected visually by at least two reviewers, comparing the intensity of alleles from normal and tumour DNA. Absence or significant decrease of one allele in the tumour compared with the normal reference sample was considered as $\mathrm{LOH}$.

\subsection{Statistical analysis}

The Chi square analysis and Fisher's exact test were used for comparison of numbers in each group and the Student's $t$-test was used for comparing mean values. Two primary clinical endpoints were looked at in this study; death from breast cancer and disease recurrence. Follow-up was until the time of the last follow-up visit or the diagnosis of recurrent disease or death, whichever came first. Disease recurrence was considered evident when either local or distal metastases were diagnosed. Data on survival were censored if the patient was still alive and without evidence of recurrent disease at the time of the last follow-up visit or at the time of death from other causes. Survival curves were constructed according to the Kaplan-Meier method [25]. Univariate comparisons of survival for all breast cancer patients and for lymph node-negative patients were conducted on the basis of E-cadherin status with a log-rank analysis. The 5 patients with in situ ductal carcinomas, 1 with tubular, 3 with mucinous carcinomas and 1 patient with infiltrating ductal carcinoma, who had evidence of distant metastases at time of diagnosis, were excluded from survival curve evaluation. Information on clinical follow-up for 2 patients with infiltrating ductal carcinomas was lacking. The Cox proportional-hazards model [26] was used in the multivariate analysis to assess the contribution of the following covariates; age of patient, lymph node status, tumour size and oestrogen receptor status. These covariates were retained in the model to illustrate the independent effect of E-cadherin status. A $P$ value of less than 0.05 was considered statistically significant.

\section{Results}

\subsection{Characteristics of patients}

Table 2 shows the relevant clinical characteristics of the 120 breast cancer patients analysed for E-cadherin expression. They were grouped by immunohistochemical E-cadherin expression into $27(23 \%)$ patients with negative staining and $93(78 \%)$ patients with evident Ecadherin staining of tumours (either positive or heterogeneous). There was no significant difference between age or follow-up of the positive and heterogeneous Ecadherin staining groups on the one hand and the negative staining group on the other. In addition, the two E-cadherin staining groups in Table 2 did not differ significantly with respect to axillary node status, oestrogen receptor expression or tumour size.

\subsection{E-cadherin expression in primary breast cancer and lymph node metastases according to histopathology}

In all, 120 paraffin sections from primary breast carcinomas and 37 lymph node metastases were stained 
Table 2

Characteristics of 120 breast cancer patients and tumours that were evaluated for E-cadherin expression ${ }^{\mathrm{a}}$

\begin{tabular}{|c|c|c|c|}
\hline \multirow[b]{2}{*}{ Characteristics } & \multirow[b]{2}{*}{ Total } & \multicolumn{2}{|l|}{ E-cadherin staining groups } \\
\hline & & Positive and heterogeneous & Negative \\
\hline Total $(\%)$ & & $93(78)$ & $27(23)$ \\
\hline Median age at diagnosis, year (range) & $58(29-92)$ & $59.2(29-92)$ & $57.1(29-83)$ \\
\hline Median follow-up, months (range) & $71(3-108)$ & $71(3-108)$ & $71(12-107)$ \\
\hline \multicolumn{4}{|c|}{ Axillary node status (117 samples informative) } \\
\hline Node-negative, $n(\%)$ & $49(42)$ & $37(41)$ & $12(46)$ \\
\hline Node-positive, $n(\%)$ & $68(58)$ & $54(59)$ & $14(54)$ \\
\hline \multicolumn{4}{|l|}{ Tumour size ( 95 samples informative) } \\
\hline$\leqslant 5 \mathrm{~cm}(\%)$ & $82(86)$ & $62(85)$ & $20(91)$ \\
\hline$>5 \mathrm{~cm}(\%)$ & $13(14)$ & $11(15)$ & $2(9)$ \\
\hline \multicolumn{4}{|c|}{ Oestrogen receptor expression (105 samples informative) } \\
\hline Oestrogen-positive, $n(\%)$ & $71(68)$ & $55(67)$ & $16(70)$ \\
\hline Oestrogen-negative, $n(\%)$ & $34(32)$ & $27(33)$ & $7(30)$ \\
\hline
\end{tabular}

a None of the differences between the two E-cadherin groups were statistically significant.

Table 3

E-cadherin staining according to histopathology (number of tumours)

\begin{tabular}{|c|c|c|c|c|}
\hline \multirow[b]{3}{*}{ Histopathology of primary breast cancer: } & \multicolumn{3}{|c|}{ E-cadherin staining groups } & \multirow[b]{2}{*}{ Total } \\
\hline & Positive & Heterogeneous & Negative & \\
\hline & $n(\%)$ & $n(\%)$ & $n(\%)$ & $n(\%)$ \\
\hline In situ ductal carcinoma (non-comedo type) & $1(33)$ & $2(67)$ & - & $3(3)$ \\
\hline In situ ductal carcinoma (comedo type) & & $2(100)$ & - & $2(2)$ \\
\hline Infiltrating ductal carcinoma NOS & $24(25)$ & $55(57)$ & $18(19)$ & $97(81)$ \\
\hline Infiltrating lobular carcinoma & $1(7)$ & $4(29)$ & $9(64)$ & $14(12)$ \\
\hline Tubular carcinoma & $1(100)$ & - & - & $1(1)$ \\
\hline Mucinous carcinoma & - & $3(100)$ & - & $3(3)$ \\
\hline Total & $27(23)$ & $66(55)$ & $27(23)$ & $120(100)$ \\
\hline Lymph node metastases & $9(24)$ & $20(54)$ & $8(22)$ & $37(100)$ \\
\hline
\end{tabular}

with monoclonal antibodies to E-cadherin. Table 3 shows E-cadherin expression according to histological type of tumours.

Histologically, $97(81 \%)$ tumours were infiltrating ductal carcinomas, $14(12 \%)$ lobular carcinomas, 3 (3\%) mucinous, $1(1 \%)$ tubular, $5(4 \%)$ in situ ductal; thereof 2 of the comedo type. In Table 3, E-cadherin staining is grouped according to histopathological type of tumour. As Table 3 shows, 73 out of 97 (75\%) of the infiltrating ductal carcinomas show a moderate to a substantial decrease in E-cadherin expression (heterogeneous or negative staining). It should be noted that complete loss of E-cadherin expression was significantly more frequent in lobular carcinoma as 64\% (9/14) showed negative staining compared with $19 \%$ of the infiltrating ductal carcinomas $(18 / 97) \quad(P=<0.001)$ although the small numbers in the former group $(n=14)$ compared with the latter group $(n=97)$ should be noted. Furthermore, the lobular carcinomas often showed a staining pattern that was not seen in other types, namely cytoplasmic staining instead of membrane staining. This staining pattern was classified as negative. Fig. 1 shows examples of the staining patterns observed.
In 37 samples (Table 4), the lymph node metastases were available for staining of E-cadherin expression. In $25(68 \%)$, E-cadherin expression was identical to the expression of their primary tumours.

\subsection{Association of E-cadherin expression with $\mathrm{LOH}$}

Of the 86 samples evaluated for loss of heterozygosity (LOH) with three markers on chromosome 16q close to the E-cadherin gene, 61 samples were informative. $\mathrm{LOH}$

Table 4

Comparison of E-cadherin staining of primary tumour and lymph node metastases $(n=37)$

\begin{tabular}{llll}
\hline & \multicolumn{2}{l}{$\begin{array}{l}\text { Primary tumour } \\
\text { E-cadherin staining groups }\end{array}$} \\
\cline { 2 - 4 } & Positive & Heterogeneous & Negative \\
\hline Lymph node metastases & & & \\
E-cadherin staining groups & & 2 & 2 \\
Positive & 5 & 14 & 2 \\
Heterogeneous & 4 & 2 & 6 \\
Negative & 0 & & \\
\hline
\end{tabular}


was detected in $52 \%(32 / 61)$ of these samples but was clearly more common in the infiltrating lobular carcinomas $(89 \% ; 8 / 9$ informative samples) (Table 5). In the infiltrating lobular carcinomas, we observed that $\mathrm{LOH}$ was most commonly associated with complete loss of Ecadherin expression (6/8 samples), although the sample group was too small for statistical analysis. Such a tendency was not seen in the infiltrating ductal carcinomas, where $\mathrm{LOH}$ was more commonly associated with heterogeneous rather than complete loss of E-cadherin expression $(71 \%, 17 / 24$ samples). This heterogeneous type of E-cadherin expression was also seen in $43 \%(12 /$ 28) of samples without LOH. Nevertheless, 3 out of 24
$(13 \%)$ infiltrating ductal carcinomas with LOH retained strong positive E-cadherin staining, whereas 5 out of 28 $(18 \%)$ without $\mathrm{LOH}$ were E-cadherin negative.

In the infiltrating lobular carcinomas, a cytoplasmic staining pattern was seen in many cells in all of the six samples that showed both $\mathrm{LOH}$ and loss of E-cadherin membrane expression on immunohistochemistry (Fig. 1). In contrast, cytoplasmic staining was never detected in the infiltrating ductal carcinomas (Fig. 1).

Two of the infiltrating lobular carcinomas that showed cytoplasmic staining and $\mathrm{LOH}$, one that showed cytoplasmic staining where PCR was non-informative and one that showed heterogeneous staining without
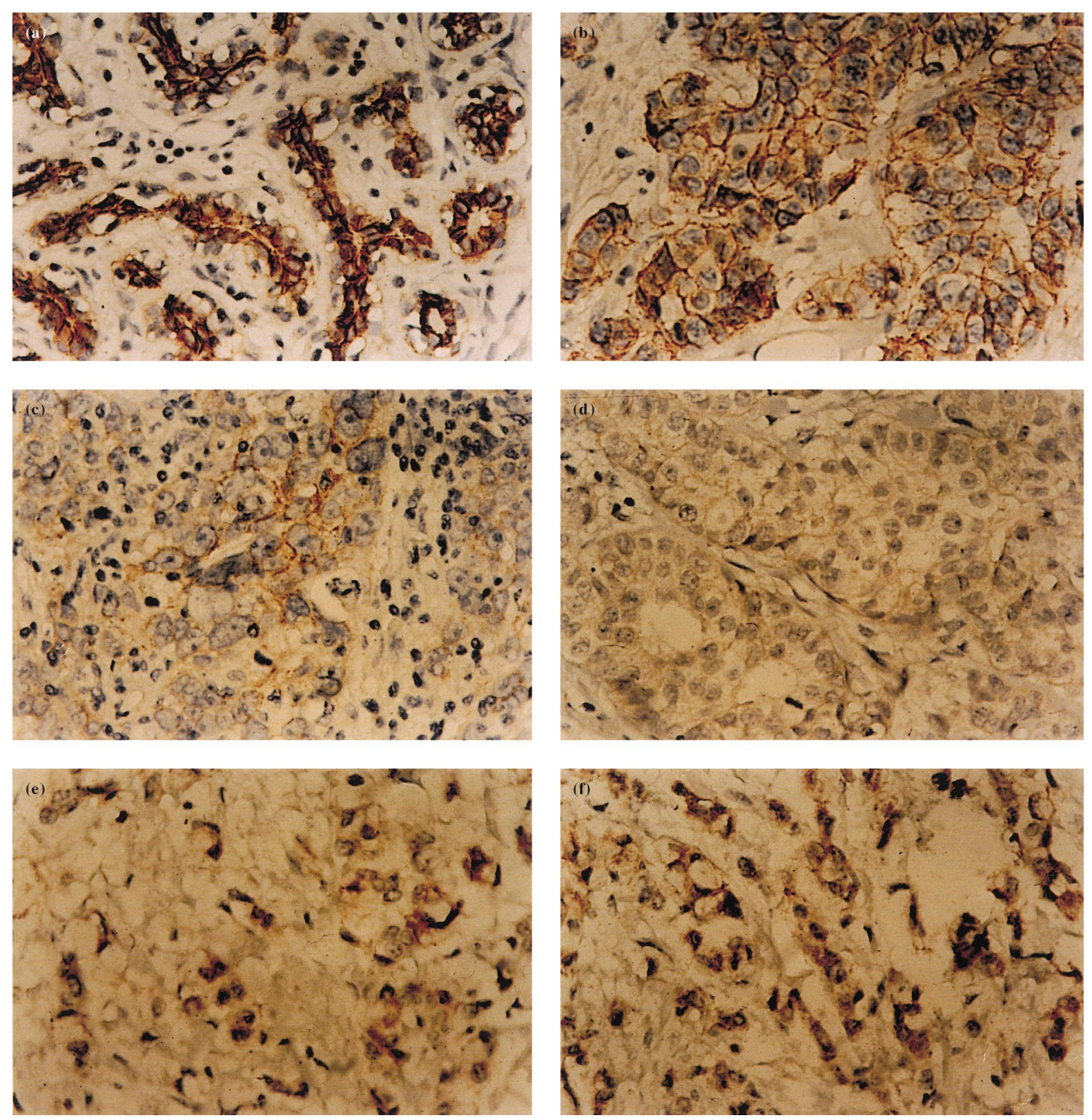

Fig. 1. Patterns of E-cadherin expression in breast carcinoma. (a) Shows a fibroadenoma sample which was used as a positive control; (b) represents an infiltrating ductal breast carcinoma sample showing positive E-cadherin expression; whereas sample (c) shows heterogeneous expression; and sample (d) is negative. Lobular breast carcinomas with cytoplasmic E-cadherin expression are shown in (e) and (f). Original magnification $\times 600$. 
Table 5

Association of E-cadherin with LOH ( $n=61$ informative $)^{\mathrm{a}}$

\begin{tabular}{|c|c|c|c|c|c|}
\hline & & \multicolumn{3}{|c|}{ E-cadherin staining groups } & \multirow[b]{2}{*}{ Total } \\
\hline & & Positive & Heterogeneous & Negative & \\
\hline \multirow[t]{2}{*}{ Infiltrating ductal carcinomas } & $+\mathrm{LOH}$ & $3(13)$ & $17(71)$ & $4(17)$ & $24(46)$ \\
\hline & $-\mathrm{LOH}$ & $11(39)$ & $12(43)$ & $5(18)$ & $28(54)$ \\
\hline \multirow[t]{2}{*}{ Infiltrating lobular carcinomas } & $+\mathrm{LOH}$ & 0 & $2(25)$ & $6(75)$ & $8(89)$ \\
\hline & $-\mathrm{LOH}$ & 0 & $1(100)$ & 0 & $1(11)$ \\
\hline
\end{tabular}

a LOH, loss of heterozygosity.

$\mathrm{LOH}$, were available for SSCP analysis and DNA sequencing for E-cadherin gene mutations. One tumour with cytoplasmic staining and $\mathrm{LOH}$ was found to have a somatic mutation at the exon 13-intron 13 boundary. The mutation was a base substitution in the first nucleotide in intron 13 from $\mathrm{G}$ to $\mathrm{A}$.

\subsection{Association of E-cadherin expression with clinical parameters, disease recurrence and survival}

Patient records on local disease recurrence, development of metastases and breast cancer-corrected survival were available for $108(90 \%)$ of the 120 patients. As shown in Fig. 2a, a trend towards a shorter disease-free survival was seen in patients having tumours with complete loss of E-cadherin expression $(P=0.08)$. When axillary node-negative patients were analysed separately, this trend nearly reached significance $(P=0.06)$ (Fig. 2b). Of the 11 E-cadherin negative/node-negative patients, 3 were infiltrating lobular- and 8 were infiltrating ductal carcinomas. When known prognostic factors, such as age of patients, lymph node status, tumour size and oestrogen hormone receptor expression were included in the multivariate Cox analysis, loss of E-cadherin expression was shown to be a significant independent risk factor for disease recurrence $(P=0.019)$ and approaching significance for breast cancercorrected survival $(P=0.056)$ (Table 6$)$. This prognostic significance was furthermore evident in node-negative breast cancer patients but not in node-positive patients, when these patient groups were analysed separately (Table 6). The effects of loss of E-cadherin expression on breast cancer-corrected survival in the node-negative patients did not reach significance. Loss of E-cadherin expression showed the highest hazard ratio for diseasefree survival amongst the factors analysed for the whole group and particularly in node-negative patients and was the only factor reaching statistical significance in this latter subgroup of patients (Table 6). LOH did not have an effect on disease-free survival or breast cancercorrected survival. In the whole patient group $72 \%$ received adjuvant chemo- and/or hormonal therapy, and treatment did not differ widely with respect to Ecadherin expression, i.e. $73 \%$ and $67 \%$ received treatment in the positive/heterogeneous group and negative group-respectively. However, in the node-negative patient group, the percentage receiving therapy was generally lower $(64 \%)$ but higher in the E-cadherinnegative patients $(57 \%)$ than the E-cadherin-positive/ heterogeneous patients $(40 \%)$.

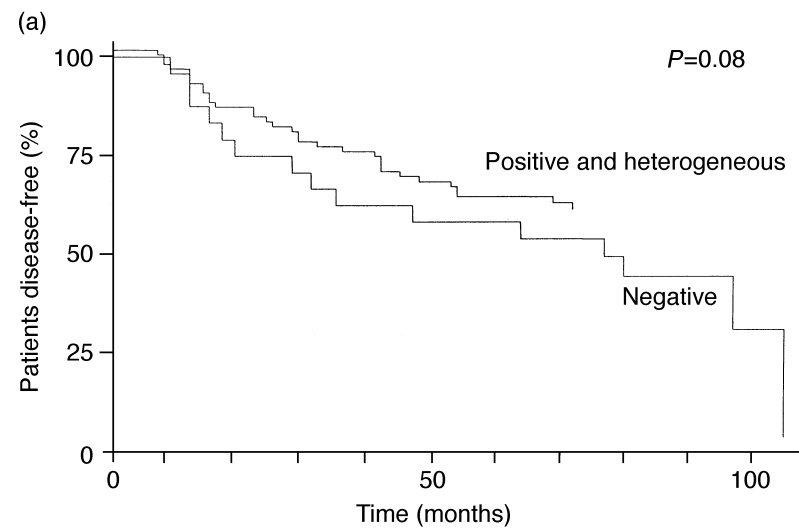

Numbers at risk :

Positive/heterogeneous 84 Negative

24

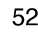

7

(b)

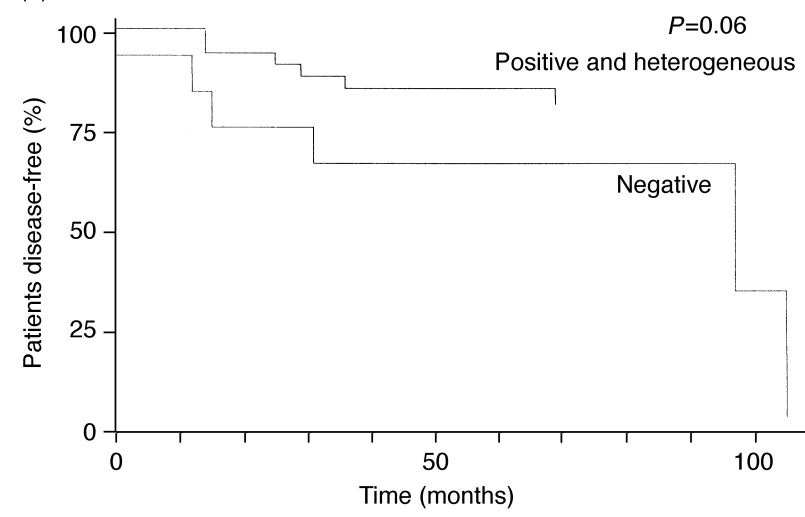

Numbers at risk :

Positive/heterogeneous 36

Negative

11

28

4

Fig. 2. Disease-free survival in relation to E-cadherin expression. Evaluation was only carried out for infiltrating ductal and lobular carcinoma cases where $n=108$ were available. The number of patients in both E-cadherin expression groups at risk are shown for all patients at 0,50 and 100 months. (a) All patients; (b) axillary lymph nodenegative patients only. 
Table 6

Cox analysis of hazard ratio estimates of individual prognostic factors

\begin{tabular}{lll}
\hline & Hazard ratio $(95 \%$ confidence interval) & $P$ value \\
\hline All patients - BCCS & & \\
Axillary lymph nodes & $2.00(0.90-4.43)$ & 0.089 \\
Tumour size & $2.36(1.31-4.26)$ & 0.004 \\
Age of patient & $1.00(0.97-1.02)$ & 0.784 \\
Negative oestrogen receptor expression & $0.70(0.33-1.49)$ & 0.353 \\
Negative E-cadherin expression & $2.20(0.98-4.96)$ \\
All patients - DFS & & 0.056 \\
Axillary lymph nodes & $2.03(0.96-4.29)$ \\
Tumour size & $1.86(1.51-2.44)$ \\
Age of patient & $0.99(0.96-1.01)$ \\
Negative oestrogen receptor expression & $1.11(1.16-5.14)$ \\
Negative E-cadherin expression & $2.44(1.12-5.14)$ \\
Node-negative patients - DFS & & 0.064 \\
Tumour size & $2.12(0.79-5.68)$ \\
Age of patient & $0.98(0.94-1.02)$ \\
Negative oestrogen receptor expression & $0.55(0.14-2.10)$ \\
Negative E-cadherin expression & $5.03(1.18-21.41)$ \\
Node-positive patients - DFS & & 0.029 \\
Tumour size & $2.93(1.23-6.98)$ \\
Age of patient & $0.99(0.95-1.03)$ \\
Negative oestrogen receptor expression & $1.71(0.54-5.44)$ \\
Negative E-cadherin expression & $1.82(0.70-4.73)$ \\
\hline
\end{tabular}

BCCS, breast cancer-corrected survival; DFS, disease-free survival.

\section{Discussion}

In this study we have shown that decreased Ecadherin expression is frequent in breast cancer and that complete loss of E-cadherin expression is associated with $\mathrm{LOH}$ in the infiltrating lobular breast carcinomas but not in the infiltrating ductal carcinomas. We also found that the infiltrating lobular breast carcinomas often show a different immunohistochemical staining pattern for E-cadherin, specifically a cytoplasmic staining pattern, which is unlike the plasma membrane pattern seen in ductal carcinomas. Finally, we found that loss of E-cadherin expression in breast cancer tumours is a significant prognostic factor. To the best of our knowledge, this is the first study to show that loss of Ecadherin expression is a significant independent risk factor for disease recurrence in axillary node-negative breast cancer patients, and in this patient group this seems to be a stronger independent prognostic factor for recurrent disease than the age of the patient, tumour size or negative oestrogen receptor expression although the $95 \%$ CIs were fairly wide.

Although many studies have shown that altered Ecadherin expression in various cancer types correlates with tumour dedifferentiation [9-12] and poor prognosis [8,13-19], the mechanisms by which these alterations occur are as yet only partially understood. Somatic mutations affecting expression of the E-cadherin gene, post-translational modifications and abnormal function of the normally expressed molecule because of altera- tions in its associated proteins, are candidate explanations, supported by both in vitro and in vivo studies [20 23,27]. Furthermore, germ line mutations in the Ecadherin gene have recently been found to occur in familial gastric cancer [28].

Studies on breast cancer have shown that LOH and mutations in the E-cadherin gene are frequently found in infiltrating lobular carcinomas but not in the infiltrating ductal subtype [29,30]. Our results support these findings. Firstly, we saw LOH on chromosome 16q22.1 in all but one of the nine infiltrating lobular carcinomas and LOH was usually, although not always, associated with complete loss of E-cadherin (6/8). The specific cytoplasmic staining pattern was in one case shown to be associated with a somatic E-cadherin gene mutation in the exon 13-intron 13 boundary. This probably results in loss of the transmembrane and cytoplasmic domains of the protein, thus affecting the correct membrane localisation. In one further sample with confirmed LOH and cytoplasmic E-cadherin expression, no mutation was found, suggesting that loss of one copy of the E-cadherin gene may affect the expression of the remaining allele.

Different characteristics were seen in infiltrating ductal breast carcinomas. LOH was not as common as in the infiltrating lobular subtype, and even when present, immunohistochemical E-cadherin expression was often evident. Furthermore, loss of E-cadherin expression could occur without LOH. It is unlikely that the immunohistochemistry failed to detect E-cadherin when present 
in samples without $\mathrm{LOH}$ since antigen retrieval and a sensitive secondary detection system were used. The discrepancies between $\mathrm{LOH}$ and immunostaining could have several explanations. A gene other than the Ecadherin gene may be the target of the 16q22.1 deletion in ductal carcinomas or the retained E-cadherin gene could possibly be functional. Mechanisms, other than gene deletions can cause loss of E-cadherin expression, including transcriptional defects and $\mathrm{CpG}$ methylation [31,32]. In vitro studies in our laboratory have shown that the cytokine IL-6 can cause a reversible decrease in E-cadherin expression in breast cancer cell lines [24], and this could provide a mechanism for dislodgement of cells from a primary tumour with re-expression as a metastasis becomes established elsewhere.

One of the drawbacks of immunohistochemical studies is that it is not a functional analysis of the molecule in question. Several studies have shown that normal immunohistochemical expression of E-cadherin can be associated with a compromised functional ability. For instance, in vitro studies have shown that defects in catenins $(\alpha, \beta, \gamma)$ which link E-cadherin to the submembrane cytoskeletal matrix, can alter the functional ability of E-cadherin [33,34]. Alterations in the expression of these proteins can be seen despite retained Ecadherin expression [23,35]. Several studies on breast cancer cell lines have shown that appropriate gene expression and post-translational modifications of these catenins must be in place for E-cadherin to be fully functional [36,37]. In MDCK cells transformed by $\mathrm{v}$-SRC transfection, the phosphorylation of the Ecadherin-catenin complex led to cell dissociation and invasiveness, although the expression of E-cadherin was retained [22].

The variable clinical outcome of breast cancer patients, has stimulated the search for new prognostic markers that have the ability to predict tumour behaviour. A long list of potential prognostic markers have now been identified [38], although the axillary lymph node status still remains the most important. For instance, the detection of mutations in the TP53 gene and immunohistochemical overexpression of the c-erbB2 protein has been correlated with a shorter overall survival of breast cancer patients $[39,40]$. The value of these new markers in the prognostic evaluation of axillary lymph node-negative breast cancer patients is, however, controversial. A prognostic panel involving the evaluation of tumour size, hormone receptor level and S-phase fraction has been shown to be able to identify those node-negative patients with a sufficiently low risk of disease recurrence so that adjuvant chemotherapy can be avoided [41].

The results of our study show that the clinical importance of E-cadherin in breast cancer depends on whether it is immunohistochemically expressed or not. In the multivariate analysis, loss of E-cadherin expression not only had an effect on breast cancer-corrected survival, but significantly increased the risk of disease recurrence in node-negative breast cancer patients. Several other studies support our findings [8,17-19]. Although they vary with respect to the types of tissue samples used (only Lipponen and colleagues used paraffinembedded samples, whereas the other authors used frozen sections), the E-cadherin antibodies and the total follow-up, most of them indicate that the overall survival of the breast cancer patients is significantly decreased if their tumours show alterations in E-cadherin expression [17-19]. Only one study has shown a correlation between altered E-cadherin expression and a decreased disease-free survival [8]. The most recent study involving 179 breast cancer patients and a 10-year follow-up shows that the effect on survival seems to be most relevant in axillary node-negative patients [18]. It is unlikely that adjuvant therapy had an impact on our survival analysis since the proportion of node-negative patients receiving therapy was generally lower $(64 \%)$ than in the group as a whole, although the E-cadherin-negative patients received more therapy $(57 \%)$ than the E-cadherin-positive patients $(40 \%)$ in this group.

Therefore, this study suggests that loss of E-cadherin expression, whether it is the result of gene mutations or other mechanisms, is an important prognostic marker, especially for disease recurrence in node-negative breast cancer patients and may even be more informative than tumour size or oestrogen receptor expression. Furthermore, immunohistochemical analysis is more informative, in this regard, than detection of $\mathrm{LOH}$ at the Ecadherin gene locus. Further studies are required in order to address the issue of whether the evaluation of E-cadherin expression can help, independently or in addition to conventional biological prognostic markers, to identify those axillary node-negative breast cancer patients who may truly benefit from adjuvant therapy.

\section{Acknowledgements}

The financial support of the Science Fund of the Icelandic Cancer Society is gratefully acknowledged. We would also like to thank the following oncologists for their help in acquiring adequate clinical information; Dr Thórarinn Sveinsson, Dr Helgi Sigurðsson and Dr Sigurður Björnsson.

\section{References}

1. Hart IR, Saini A. Biology of tumour metastasis. Lancet 1992, 339, 1453-1457.

2. Takeichi M. Cadherins: a molecular family important in selective cell-cell adhesion. Annu Rev Biochem 1990, 59, 237-252.

3. Behrens J, Birchmeier W, Goodman SL, Imhof BA. Dissociation of Madin-Darby canine kidney epithelial cells by the monoclonal 
antibody anti-arc-1: mechanistic aspects and identification of the antigen as a component related to uvomorulin. J Cell Biol 1985, 101, 1307-1315.

4. Behrens J, Mareel MM, Van Roy FM, Birchmeier W. Dissecting tumor cell invasion: epithelial cells acquire invasive properties after the loss of uvomorulin-mediated cell-cell adhesion. $J$ Cell Biol 1989, 108, 2435-2447.

5. Frixen UH, Behrens J, Sachs M, et al. E-cadherin-mediated cellcell adhesion prevents invasiveness of human carcinoma cells. $J$ Cell Biol 1991, 113, 173-185.

6. Oka H, Shiozaki H, Kobayashi K, et al. Expression of Ecadherin cell adhesion molecules in human breast cancer tissues and its relationship to metastasis. Cancer Res 1993, 53, 16961701.

7. Perl A-K, Wilgenbus P, Dahl U, Semb H, Christofori G. A causal role for E-cadherin in the transition from adenoma to carcinoma. Nature 1998, 392, 190-193.

8. Siitonen SM, Kononen JT, Helin HJ, Rantala IS, Holli KA, Isola JJ. Reduced E-cadherin expression is associated with invasiveness and unfavorable prognosis in breast cancer. Am J Clin Pathol 1996, 105, 394-402.

9. Gamallo C, Palacios J, Suarez A, et al. Correlation of E-cadherin expression with differentiation grade and histological type in breast carcinoma. Am J Pathol 1993, 142, 987-993.

10. Moll R, Mitze M, Frixen UH, Birchmeier W. Differential loss of E-cadherin expression in infiltrating ductal and lobular breast carcinomas. Am J Pathol 1993, 143, 1731-1742.

11. Yamada K, Jordan R, Mori M, Speight PM. The relationship between E-cadherin expression, clinical stage and tumour differentiation in oral squamous cell carcinoma. Oral Dis 1997, 3, 8285.

12. Mayer B, Johnson JP, Leitl F, et al. E-cadherin expression in primary and metastatic gastric cancer: down-regulation correlates with cellular dedifferentiation and glandular disintegration. Cancer Res 1993, 53, 1690-1695.

13. Katagiri A, Watanabe R, Tomita Y. E-cadherin expression in renal cell cancer and its significance in metastasis and survival. $\mathrm{Br}$ J Cancer 1995, 71, 376-379.

14. Tamura S, Shiozaki H, Miyata M, et al. Decreased E-cadherin expression is associated with haematogenous recurrence and poor prognosis in patients with squamous cell carcinoma of the oesophagus. Br J Surg 1996, 83, 1608-1614.

15. Gabbert HE, Mueller W, Schneider A, et al. Prognostic value of E-cadherin expression in 413 gastric carcinomas. Int $J$ Cancer 1996, 69, 184-189.

16. Otto T, Bex A, Schmidt U, Raz A, Rubben H. Improved prognosis assessment for patients with bladder carcinoma. Am J Pathol 1997, 150, 1919-1923.

17. Lipponen P, Saarelainen E, Aaltomaa S, Syrjanen K. Expression of E-cadherin (E-CD) as related to other prognostic factors and survival in breast cancer. J Pathol 1994, 174, 101-109.

18. Charpin C, Garcia S, Bonnier P, et al. Reduced E-cadherin immunohistochemical expression in node-negative breast carcinomas correlates with 10-year survival. Am J Clin Pathol 1998, 109, 431-438.

19. Gamallo C, Palacios J, Benito N, et al. Expression of E-cadherin in 230 infiltrating ductal breast carcinomas - relationship to clinicopathologic features. Int J Oncol 1996, 9, 1207-1212.

20. Becker KF, Atkinson MJ, Reich U, et al. E-cadherin gene mutations provide clues to diffuse type gastric carcinomas. Cancer Res 1994, 54, 3845-3852.

21. Berx G, Cleton-Jansen AM, Nollet F, et al. E-cadherin is a tumour/invasion suppression gene mutated in human lobular breast cancers. EMBO J 1995, 14, 6107-6115.

22. Behrens J, Vakaet L, Friis R, et al. Loss of epithelial differentiation and gain of invasiveness correlates with tyrosine phosphorylation of the E-cadherin/beta-catenin complex in cells transformed with a temperature-sensitive v-SRC gene. J Cell Biol 1993, 120, 757766.

23. Shimoyama Y, Nagafuchi A, Fujita S, et al. Cadherin dysfunction in a human cancer cell line: possible involvement of loss of alpha-catenin expression in reduced cell-cell adhesiveness. Cancer Res 1992, 52, 5770-5774.

24. Ásgeirsson KS, Ólafsdóttir K, Jónasson JG, Ögmundsdóttir HM. The effects of IL-6 on cell adhesion and E-cadherin expression in breast cancer. Cytokine 1998, 10, 720-728.

25. Kaplan FL, Meier P. Non parametric estimations from incomplete observations. J Am Stat Assoc 1958, 53, 457-481.

26. Cox DR. Regression models and life-tables. J Royal Stat Soc 1972, 34, 187-220.

27. Risinger JI, Berchuck A, Kohler MF, Boyd J. Mutations of the E-cadherin gene in human gynecologic cancers. Nature Genet 1994, 7, 98-102.

28. Guilford $\mathrm{P}$, Hopkins $\mathrm{J}$, Harraway $\mathrm{J}$, et al. E-cadherin germline mutations in familial gastric cancer. Nature 1998, 392, 402-405.

29. Huiping C, Sigurgeirsdóttir J, Jónasson JG, et al. Chromosome alterations and E-cadherin gene mutations in human lobular breast cancer. Br J Cancer 2000, 81, 1103-1128.

30. Berx G, Cleton-Jansen AM, Strumane K, et al. E-cadherin is inactivated in a majority of invasive human lobular breast cancers by truncation mutations throughout its extracellular domain. Oncogene 1996, 13, 1919-1925.

31. Ji X, Woodard AS, Rimm DL, Fearon ER. Transcriptional defects underlie loss of E-cadherin expression in breast cancer. Cell Growth Different 1997, 8, 773-778.

32. Graff JR, Greenberg VE, Herman JG, et al. Distinct patterns of E-cadherin CpG methylation in papillary, follicular, Hurthle's cells, and poorly differentiated human thyroid carcinoma. Cancer Res 1998, 58, 2063-2066.

33. Nagafuchi A, Takeichi M. Cell binding function of E-cadherin is regulated by the cytoplasmic domain. EMBO J 1988, 7, 36793684

34. Ozawa M, Ringwald M, Kemler R. Uvomorulin-catenin complex formation is regulated by a specific domain in the cytoplasmic region of the cell adhesion molecule. Proc Natl Acad Sci USA 1990, 87, 4246-4250.

35. Kadowaki $\mathrm{T}$, Shiozaki $\mathrm{H}$, Inoue $\mathrm{M}$, et al. E-cadherin and $\alpha$ catenin expression in human esophageal cancer. Cancer Res 1994 54, 291-296.

36. Sommers CL, Gelmann EP, Kemler R, Cowin P, Byers SW. Alterations in $\beta$-catenin phosphorylation and plakoglobin expression in human breast cancer cells. Cancer Res 1994, 54, 3544-3552.

37. Pierceall WE, Woodard AS, Morrow JS, Rimm D, Fearon ER. Frequent alterations in E-cadherin and $\alpha$ and $\beta$-catenin expression in human breast cancer cell lines. Oncogene 1995, 11, 13191326.

38. Norberg T, Jansson $\mathrm{T}$, Sjogren $\mathrm{S}$, et al. Overview on human breast cancer with focus on prognostic and predictive factors with special attention on the tumour suppressor gene p53. Acta Oncol 1996, 35, 96-102.

39. Sjogren S, Inganas M, Lindgren A, Holmberg L, Bergh J. Prognostic and predictive value of c-erB-2 overexpression in primary breast cancer, alone and in combination with other prognostic markers. J Clin Oncol 1998, 16, 462-469.

40. Sjogren $\mathrm{S}$, Inganas $\mathrm{M}$, Norberg $\mathrm{T}$, et al. p53 gene in breast cancer: prognostic value of complementary DNA sequencing versus immunohistochemistry. J Natl Cancer Inst 1996, 88, 173 182.

41. Sigurdsson H, Baldetorp B, Borg A, et al. Indicators of prognosis in node-negative breast cancer. $N$ Engl $J$ Med 1990, 322, 1045 1053. 